\title{
Clinical Profile of Patients Subjected for Colour Doppler of Carotid Arteries
}

\author{
S Mahbub Pasha ${ }^{1}$ \\ ${ }^{1}$ Assistant Professor, Department of Radiology, SS Institute Of Medical sciences and Research Centre, Davangere, Karnataka.
}

\section{Abstract}

Background: The management of asymptomatic carotid disease remains controversial. The finding of a cervical bruit has been used as a marker of carotid disease in asymptomatic patients and studies have suggested that these patients are at an increased risk of TIA and strokes when compared with the age matched controls. Some investigator however feel the risks of stroke is not increased in this patients group but they are at increased risk of death from cardiac diseases. Subjects and Methods: The study was carried out on 40 patients. Among them 20 were symptomatic cases suspected of cerebrovascular insufficiency were considered as cases, other 20 asymptomatic individuals with unrelated diseases having one or more risk factors for cerebrovascular disease on the basis of clinical examination and history were taken as controls. Results: Out of 10 patients (50\%) with atheromatous plaque in carotid bifurcation 3 had on right side (30\%), 4 on left side (40\%) and 3 on both sides (30\%). Among the 4 patients (20\%) with atheromatous plaque in common carotid artery, 2 on the right side (50\%) and 2 on left side (50\%).In 6 patients (30\%) with atheromatous plaque in internal carotid artery, $1(16.6 \%)$ had on the right side, 2 on the left side (33.3\%) and 3 on both sides (50\%). Conclusion: Among the 40 individuals, 20 were symptomatic cases suspected of cerebrovascular insufficiency and the other 20 were taken as controls with unrelated diseases having one or more risk factors for cerebrovascular disease. The incidence of male to female ratio of atherosclerotic disease of extracranial carotid system among cases was 3:2, while it was 7:3 among the controls.

Keywords: TIA, Common Carotid Artery, Colour Doppler.

Corresponding Author: Dr. S Mahbub Pasha, Assistant Professor, Department of Radiology, SS Institute Of Medical sciences and Research Centre, Davangere, Karnataka

Received: June 2019

Accepted: June 2019

\section{Introduction}

Symptomatic patients with hemispheric symptoms including those with amaurosis fugax, transient ischemic attack (TIA) and ischemic strokes are advised cerebral angiography as soon as possible, when otherwise healthy patients have TIA traceable to the carotid artery49. These patients have a high risk of stroke particularly in the first three months after onset of symptoms. Only about one half will be found to have carotid stenosis of more than $50 \%$ on the symptomatic side are good candidates for carotid endarterectomy. Non invasive carotid evaluation may increase the sense of urgency in such cases if it demonstrates critical (more than 75\%) narrowing of the artery. $^{[1]}$

In patients considered as borderline candidates for surgery because of either advanced age or a systemic medical illness such as heart failure, angiography and surgery might be reconsidered if a critical cervical carotid stenosis were demonstrated. The demonstration of significant carotid disease by Doppler evaluation may also be a deciding factor in undertaking further cerebrovascular investigations when the diagnosis of TIA is uncertain. Ultrasound evaluation of carotid system is rarely of the view when clinical examination and CT scanning indicate cerebral infarction, since the discovery of even significant carotid disease is unlikely to lead to further investigations. In patients showing good recovery from their stroke the demonstration of critical carotid stenosis may suggest the need for further investigation and for carotid endarterectomy to prevent further strokes. ${ }^{[2]}$

Another valuable use of Duplex scanning in symptomatic patient is to determine what type of angiogram to perform. If the Duplex scan shows a high grade stenosis at the carotid bifurcation, this can be easily assessed by intra-arterial digital substraction angiography (DSA). If the Duplex scan shows minimal to no disease, the appropriate investigation is a standard angiogram. This is because in the second case the offending lesion is likely to be either very small or at some site not accessible to Duplex examination.

The management of asymptomatic carotid disease remains controversial. The finding of a cervical bruit has been used as a marker of carotid disease in asymptomatic patients and studies have suggested that these patients are at an increased risk of TIA and strokes when compared with the age matched controls. Some investigator however feel the risks of stroke is not increased in this patients group but they are at increased risk of death from cardiac diseases. It is 
therefore argued that non-operative management of asymptomatic carotid lesion is the preferred approach. ${ }^{[3]}$

One reason why this problem is so unclear is that a cervical bruit is a poor marker of carotid stenosis. Bruit clearly occurs commonly in patients without carotid stenosis. Moreover, not all patients with carotid stenosis have cervical bruits. Fienberg et al showed that $73 \%$ of the patients with documented carotid stenosis on angiography did not have a bruit.

Taylor and Strandness found that a stenosis reducing vessel diameter by greater than $80 \%$ was associated with a $35 \%$ risk of developing symptoms of ipsilateral occlusion within 16 months and a $46 \%$ risk at 12 months. Conversely, only $12.1 .5 \%$ of the lesions that remained in the less than $80 \%$ category produced a complication. Thus, on this basis they now recommend carotid endarterectomy to those patients with an asymptomatic carotid stenosis of greater than $80 \%$ diameter reduction if they are good operative candidates. On the other hand, it is recommended that it is safe to observe patients with stenosis less than $80 \%$. Thus, they have identified by Duplex scanning a group of patients having asymptomatic carotid stenosis who are at risk of cerebrovascular complications. ${ }^{[4]}$

These results in patients with asymptomatic carotid stenosis have been further substantiated by Baird who found that asymptomatic patients with greater than $75 \%$ of stenosis detected by Duplex scanning went on to suffer unheralded strokel. He also found that patient with asymptomatic carotid stenosis less than $75 \%$ had a low risk for stroke. Bogousslava et al followed 38 patients with asymptomatic carotid stenosis greater than $90 \%$ by Duplex scanning for a mean period of 4 years. He found that $50 \%$ suffered an adverse neurological deficit in follow-up (TIA, stroke or occlusion).

These data show that Duplex scanning in patient with asymptomatic carotid disease discovers a group at high risk for adverse cerebrovascular events. With the widespread use of this technology more and more patients with asymptomatic carotid disease will be identified. The Doppler scanner has a major role to play in the management of these patients.

Duplex scanning can define the natural history of the endarterectomized carotid artery. On clinical symptoms the rate of recurrence of carotid stenosis is less than $5 \%$. However, when Duplex scanning became widely used on patients following endarterectomy, it became evident that the incidence of restenosis was much high. Ziegler et al showed an initial restenosis rate of $36 \%$ with subsequent regression in many and a persistent restenosis rate of $19 \%$. Majorities of these lesions were asymptomatic. These unexpected findings have been confirmed by others. Particularly interesting is the generally benign course followed by the lesions in that they tend not to progress to symptoms or occlusion and infarct often regresses in early postoperative period. These lesions are not atherosclerotic but are related to smooth muscle proliferation in the endarterectomized vessel. Follow up of these lesions with Duplex scanning will provide information regarding the process of arterial healing and interventional strategies designed to reduce its incidence. ${ }^{[5]}$
Duplex ultrasound is an ideal research tool. It is noninvasive, accurate and reasonably inexpensive. It will continue to have a major role in better defining the natural history of atherosclerotic lesions of the extracranial arteries in both symptomatic and asymptomatic patients. The effect of non-surgical intervention to control atherosclerosis such as control of hypertension can be monitored.

\section{Subjects and Methods}

The life of an individual is often dramatically affected by having a stroke. Not only is stroke a leading cause of adult disability but also death. The direct and indirect costs associated with strokes are enormous.

The main role of Duplex Carotid examination in carotid artery is the detection of occlusive lesions in the vicinity of carotid bifurcation, because these are the patients who carry increased risk of cerebral infarction. So carotid Doppler evaluation is a cheap, simple and safe method for assessment of stenosis of the cervical portions of carotid artery.

Study Setting: This study was carried out at Teaching \& General Hospital and Government General Hospital, attached to Medical College.

Study Duration: This study was carried out for 18 months Source of Data: All clinically suspected cerebrovascular insufficiency cases referred for colour Doppler of carotid arteries to the Radiology Department

Study Population: The study was carried out on 40 patients. Among them 20 were symptomatic cases suspected of cerebrovascular insufficiency were considered as cases, other 20 asymptomatic individuals with unrelated diseases having one or more risk factors for cerebrovascular disease on the basis of clinical examination and history were taken as controls.

Inclusion Criteria: All cases referred to the Radiology Department with history and clinical findings consistent with cerebrovascular insufficiency for Colour Doppler of carotid arteries.

Exclusion Criteria: 1. Symptoms suggestive of vertebrobasilar insufficiency. 2. All cases of head injuries. 3. Cases of primary and metastatic brain tumors.

Method of Collection of Data: The proforma was designed based on the objective of the study and it was pretested and used after modification.

Detailed clinical history and central nervous system examination, findings were noted. Evidence of hypertension, diabetes mellitus, hyperlipidemia and ischemic heart disease were collected. The patients selected as per inclusion criteria were subjected to carotid Doppler examination.

The data gathered from the carotid Doppler
examination were as follows:
Peak systolic velocity (PSV) of common carotid artery
(CCA) Peak systolic velocity (PSV) of internal carotid
artery (ICA). Peak systolic velocity ratio between internal
carotid artery and common carotid artery. Measurement of
vesscl lumen was donc from frozen rcal-timc image, the
plaque characteristics were seen on real-time image. e




\section{Pasha; Clinical Prafile of Patients Suljected far Calour Dappler of Carrotid Arteries}

Presence of spectral broadening or turbulence were noted down.

\section{Results}

The study was carried out on 40 patients, 20 were symptomatic cases suspected of cerebrovascular insufficiency and other 20 individuals were asymptomatic or admitted in wards with unrelated diseases but having one or more risk factors for cerebrovascular disease. On the basis of history, clinical examination and laboratory reports were taken as controls. All the cases and controls were subjected to carotid Doppler study.

Table 1: Age and sex-wise distribution of cases and control groups

\begin{tabular}{|c|c|c|c|c|c|c|c|c|}
\hline \multirow{3}{*}{$\begin{array}{l}\text { Age } \\
\text { (years) }\end{array}$} & \multicolumn{4}{|c|}{ Cases } & \multicolumn{4}{|c|}{ Controls } \\
\hline & \multicolumn{2}{|c|}{ Males $(n=12)$} & \multicolumn{2}{|c|}{ Females $(n=8)$} & \multicolumn{2}{|c|}{ Male $(n=14)$} & \multicolumn{2}{|c|}{ Female $(n=6)$} \\
\hline & No. & Percent & No. & Percent & No. & Percent & No. & Percent \\
\hline$<40$ & 0 & 0 & 0 & 0 & 1 & 7.1 & 0 & 0 \\
\hline $40-50$ & 1 & 8.3 & 0 & 0 & 2 & 14.2 & 0 & 0 \\
\hline $50-60$ & 4 & 33.3 & 0 & 0 & 3 & 21.4 & 1 & 16.6 \\
\hline $60-70$ & 5 & 41.6 & 3 & 37.5 & 5 & 35.7 & 0 & 0 \\
\hline $70-80$ & 1 & 8.3 & 4 & 50 & 2 & 14.2 & 3 & 50.0 \\
\hline$>80$ & 1 & 8.3 & 1 & 12.5 & 1 & 7.1 & 2 & 33.3 \\
\hline
\end{tabular}

Table 2: Symptoms-wise distribution of cases

\begin{tabular}{|c|c|c|c|c|c|c|}
\hline \multirow[t]{2}{*}{ Symptoms } & \multicolumn{2}{|c|}{ Males $(n=12)$} & \multicolumn{2}{|c|}{ Females $(n=8)$} & \multicolumn{2}{|c|}{ Total $(n=20)$} \\
\hline & No. & Percent & No. & Percent & No. & Percent \\
\hline Hemipaxesis & 4 & 30.0 & 3 & 42.0 & 7 & 35.0 \\
\hline Hemisensory disturbances & 1 & 7.6 & 2 & 14.0 & 3 & 15.0 \\
\hline Transient monocular blindness & 1 & 7.6 & 1 & 14_0 & 2 & 10.0 \\
\hline Monoparesis & 0 & 0.0 & 0 & 0.0 & 0 & 0.0 \\
\hline Aphasia, Dysealculia & 1 & 7.6 & 0 & 0.0 & 1 & 5.0 \\
\hline Hemiplegia & 3 & 23.0 & 2 & 28.0 & 5 & 25 \\
\hline Stroke & 2 & 15.3 & 0 & 0.0 & 2 & 10.0 \\
\hline
\end{tabular}

In the cases group $(n=20), 12$ patients were male $(60 \%)$ and 8 patients were female $(40 \%)$.

In the control group $(n=20), 14$ were male $(70 \%)$ and 6 were female $(30 \%)$.

In the cases group majority of the male patients were in the age group of 60 - 70 years age group $(41.6 \%)$ followed by the age group 50-60 years $(33.3 \%)$ and $1(8.3 \%)$ each in the age group of 40-50 years, 70-80 years and years.

In the cases group, majority of the female patients were in the age group of $70-80$ years $(50 \%)$ and the least in ?.-80 years age group $(12.5 \%)$.

In the control group $(n=20)$, most cases of males were in the age group of $60-70$ years $(35.7 \%)$ followed by $50-60$ years age group (21.4\%).

Among cases (n 20), hemiparesis was the most common presenting symptom 7 (35\%), followed by hemiplegia 5 $(25 \%)$, hemisensory disturbances $3(15 \%)$, transient monocular blindness $2(10 \%)$, stroke 2 (10\%), Aphasia, Dyscaiculia $1(5 \%)$.

Table 3: Risk factor wise distribution of cases and control groups

Table 3: Risk factor wise distribution of cases and control groups
\begin{tabular}{|l|l|l|l|l|}
\hline \multirow{2}{*}{ Risk factors } & Cases (n=20) & Controls (n=20) \\
\cline { 2 - 6 } & Number & Percent & Number & Percent \\
\hline Diabetes mellitus & 10 & 50.0 & 8 & 40.0 \\
\hline Hypertension & 11 & 55.0 & 8 & 40.0 \\
\hline Hyperlipidemia & 10 & 50.0 & 5 & 25.0 \\
\hline Smoking > 10 years & 12 & 60.0 & 8 & 40.0 \\
\hline Proved ischemic heart disease & 3 & 15.0 & 2 & 10.0 \\
\hline
\end{tabular}

Table 4: Distribution of atberoinatous plaques in cases and controls

\begin{tabular}{|l|l|l|l|c|}
\hline \multirow{2}{*}{ Peak systolic velocity ratio of ICA/ CCA } & \multicolumn{2}{l|}{ Cases (n20) } & \multicolumn{3}{l|}{ Controls (n=20) } \\
\cline { 2 - 6 } & Number & Percent & Number & Percent \\
\hline$<1.5$ & 8 & 40.0 & 9 & 45.0 \\
\hline$>1.5$ & 5 & 25.0 & 10 & 50.0 \\
\hline$>1.8$ & 3 & 15.0 & 1 & 5.0 \\
\hline
\end{tabular}


hypertension 11 (55\%), diabetes mellitus 11 (50\%), hyperlipidemia $10(50 \%)$, proved ischemic heart disease in $3(15 \%)$.

In the control group, most commonly found risk factors were diabetes mellitus in $8(40 \%)$, hypertension in $8(40 \%)$, smoking in $8(40 \%)$ followed by hyperlipidemia in $5(25 \%)$ cases and proved ischemic heart disease in $2(10 \%)$ cases.
In the cases group, atheromatous plaques was most commonly found on left side $8(40 \%)$ cases followed by right $6(30 \%)$ and bilateral i.e., in both the carotids in 6 cases $(30 \%)$.

In the control group, atheromatous plaque was most commonly found on left side $10(50 \%)$ cases, followed by right side in $9(45 \%)$ and bilateral in 1 patient $(5 \%)$.

Table 5: Distribution of atheromatous plaque in cases according to site

\begin{tabular}{|c|c|c|c|c|c|c|c|c|c|c|}
\hline \multirow[t]{2}{*}{ Plaque Site } & \multicolumn{2}{|c|}{ i. ifurcation $(n=10)$} & \multicolumn{2}{|c|}{$\operatorname{CCA}(n=4)$} & \multicolumn{2}{|c|}{ ICA $(n=6)$} & \multicolumn{2}{|c|}{$\operatorname{ECA}(n=0)$} & \multirow{2}{*}{\multicolumn{2}{|c|}{ Total $(n=20)$}} \\
\hline & No. & $\%$ & No. & $\%$ & No. & $\%$ & No. & $\%$ & & \\
\hline Right & 3 & 30 & 2 & 50 & 1 & 16.6 & 0 & 0 & 6 & 30 \\
\hline Left & 4 & 40 & 2 & 50 & 2 & 33.3 & 0 & 50 & 8 & 40 \\
\hline Bilateral & 3 & 30 & 0 & 0 & 3 & 50 & 0 & 0 & 6 & 30 \\
\hline
\end{tabular}

Out of 10 patients $(50 \%)$ with atheromatous plaque in carotid bifurcation 3 had on right side (30\%), 4 on left side $(40 \%)$ and 3 on both sides (30\%).

Among the 4 patients $(20 \%)$ with atheromatous plaque in common carotid artery, 2 on the right side $(50 \%)$ and 2 on left side $(50 \%)$.

In 6 patients $(30 \%)$ with atheromatous plaque in internal carotid artery, $1(16.6 \%)$ had on the right side, 2 on the left side $(33.3 \%)$ and 3 on both sides $(50 \%)$.

None of the patients had plaque in the external carotid artery.

\section{Discussion}

Cerebrovascular disease is a major killer today, behind only malignancy and cardiovascular diseases, as per American Statistics studies in the past have indicated that there is an unusually high prevalence of atherosclerotic disease, especially cerebrovascular disease and cardiovascular disease, in Indians inspite of the fact that the prevalence of traditional risk factors for atherosclerosis is low. This paradox has been proved by several studies on Indian immigrant population in USA, Great Britain, Australia and other European countries.

The main role of carotid Doppler examination in the carotid artery disease is the detection of occlusive lesions in the vicinity of carotid bifurcation and internal carotid artery (ICA). The accurate diagnosis of critical stenosis (more or equal to $80 \%$ decrease in diameter) is important because these are the patients who carry increased risk of cerebral infarction.

Also important clinically are those patients with lesser degrees of stenosis, as cerebral embolism may result from thrombosis of these vessels according to Chambers BR, Norris JW (1986) and Caplan LR (1986). Moreta GL, Taylor DC, Nicholls L et al (1987) have noted that the carotid lesions with less than $70 \%$ stenosis are generally treated medically with drugs that inhibit platelet aggregation and thrombus formation. ${ }^{[6]}$

The North American Symptomatic Carotid Endarterectomy Trial (NASCET) study, and the Asymptomatic Carotid Atherosclerosis Study (ACAS) as quoted by Michelle L Robbin, Mark E et al (1997) have demonstrated that the surgical treatment of endarterectomy should be reserved for those patients with carotid stenosis of more than $70 \%$. Angiography as the 'Gold Standard' has amply been replaced by Duplex ultrasonogaphy in most diagnostic circumstances. Positive experience with carotid Duplex ultrasonography scanning unassisted by angiography in the setting of an impending surgical intervention has been embraced by a growing number of doctors. ${ }^{[7]}$

So the present study was done to evaluate extracranial carotid arterial system by carotid Doppler in the population who presented with cerebrovascular insufficiency and comparing them with asymptomatic individuals taken as controls.

In the 20 cases studied, 12 patients were male (60\%) and 8 patients were female $(40 \%)$. In the control group of 20,14 individuals were male (70\%) and 6 were females (30\%).

In the cases group, majority of the male patients were in the age group of $60-70$ years $(41 \%)$ and $37 \%$ of female patients were in the age group of 70-80. In the controls group, majority of the males were in $60-70$ years $(35 \%)$ and female were in the age of $70-80$ years $(50 \%)$.

These finding correlated well with both the NASCET and the ACAS studies, where NASCET had a mean age of 8 years with $72 \%$ male and $28 \%$ female patients. The ACAS study had $50 \%$ of patients above the age group of 60 years. ${ }^{[8]}$

In our study out of 20 cases, 12 were males and 8 were females with a ratio of $3: 2$. This is comparable to the study of Paiv ansalo M, Leinonen S, Turunen I et al (1996), where male to female ratio was $4: 2$. Among the controls, 14 were males and 6 were females with a ratio of 7:3.

The traditional risk factors for stroke/ TIA include diabetes mellitus, hypertension, smoking, hyperlipidemia and coronary artery diseases. ${ }^{[9]}$

We found in our study of cases $(n=20)$, that cigarette smoking was the commonest risk factor $(60 \%)$, followed by hypertension (55\%), diabetes mellitus (50\%) and hyperlipidemia (50\%) and ischemic heart disease (15\%).

In the control group $(\mathrm{n}=20)$, the most commonly found risk factor were diabetes mellitus (40\%), smoking (40\%) and hypertension $(40 \%)$ and the least common was ischemic heart disease $(10 \%)$. These findings correlated well with the established studies.

Whisnant JP et al (1990) reported cigarette smoking of long duration to be the strongest predictor of severe extracranial 


\section{Pasha; Clinical Prafile of Patients Suljected far Calour Dappler of Caratid Arteries}

carotid atherosclerosis. Toshifumi Mannami did a study on prevalence of asymptomatic carotid atherosclerotic lesions detected by ultrasonography and its relationship to cardiovascular risk factors in the urban population and concluded that there are strong relationship between most of the established cardiovascular risk factors and carotid atherosclerosis lesion. ${ }^{[10]}$

George Howard showed that most risk factors continue to be associated with increased atherosclerosis at older ages, possibly suggesting a continued value in investigation of strategies to reduce atherosclerosis by reducing risk factors at older age.

In our study of cases atheromatous plaque was most commonly found on left side in 8 patients $(40 \%)$ on right side in 6 patients $(30 \%)$ and bilateral in 6 patients $(30 \%)$. In the control group, plaque was most commonly found on left side in $10(50 \%)$ followed by right side in $9(45 \%)$ and bilateral in $1(5 \%)$. 10 patients $(50 \%)$ had atheromatous plaque in carotid bifurcation, 3 on right side (30\%), 4 on left side $(40 \%)$ and 3 on both sides $(30 \%)$.

\section{Conclusion}

Among the cases, the highest incidence of cerebrovascular insufficiency was noted in the males (41\%) in the age group $60-70$ years and in females $37 \%$ in the age (70-80 years), while in the control group it was $35 \%$ males in the age of $60-70$ years and $50 \%$ females in the age group of $70-80$ years. Among cases, cigarette smoking was the most common risk factor constituting $60 \%$ associated with stroke/ TIA followed by hypertension in $55 \%$, hyperlipidemia and diabetes in 50\% each and ischemic heart disease in $15 \%$ of the cases respectively, while in the control group diabetes mellitus, hypertension, smoking constituted $40 \%$ each and the least was ischemic heart disease (10\%). Among the cases, atheromatous plaque was the most commonly found on the left side (40\%) followed by $30 \%$ each on right side and bilateral, while in the controls, it was most common on the left side (50\%) followed by right $(45 \%)$ and bilateral $(5 \%)$. Among the cases, the most common site for atheromatous plaque was carotid bifurcation (50\%) and the least was observed in 30\% each on right and both sides. e In common carotid artery, $50 \%$ had atheromatous plaque on right side and other $50 \%$ on left side. In internal carotid artery, atheromatous plaque was found more in left ICA (33\%), right side (16\%) and both sides $(50 \%)$.

\section{References}

1. Sabcti, Schillinger, Mlckush et al. Quantification of internal carotid artery stenosis with duplex US. Radiology 2004; Vol. 232: 431-437.

2. Zwiebel. Introduction to vascular ultrasonography. 4th Edn. Saunders Company, 2000.

3. Kenneth JW, Taylor. Clinical applications of Doppler ultrasound, 2nd Ed., Lippincott-Raven, 1995; 99-107.

4. Magyar T et al. Extreme hyperlipidemia is associated with increased IMT of CCA in patients below 55 years. European Journal of Surgery 9 (Suppl-2): 105-161.

5. Enevoldsen EM et al. Outcome for patients with carotid stenosis undergoing endarterectomy, the cerebral condition followed by extra intracranial ultrasound examination. Acta Neurol Scand 1999 June; 99(6): 348-8.

6. Asymptomatic Carotid Atherosclerosis Study (ACAS) Interim Results, NEJ1V1 1999; 1163-75.

7. Davidson's Principles and Practice of Medicine, 19th Edn. Churchill Livingstone 2002; 1159-1166.

8. North American Symptomatic Carotid Endarterectomy Trial Collaborators: Beneficial effect of carotid endartectomy in symptomatic patients with high grade carotid stenosis. N Engl J Med 1991; 325: 445 .

9. Rothwell PM et al. Equivalence of measurements of carotid stenosis. A comparison of three methods on 1001 angiograms. Stroke 1994; 25: 2435-2439.

10. Pignoli $\mathrm{P}$ et al. B-mode imaging of the combined intimal plus medial thickness (IMT) did not differ significantly from the (IMT) as measured on pathological examination. Circulation 1986; 74 : $1399 \neg 1406$.

Copyright: (C) the author(s), publisher. Asian Journal of Medical Radiological Research is an Official Publication of "Society for Health Care \& Research Development". It is an open-access article distributed under the terms of the Creative Commons Attribution Non-Commercial License, which permits unrestricted non-commercial use, distribution, and reproduction in any medium, provided the original work is properly cited.

How to cite this article: Pasha SM. Clinical Profile of Patients Subjected for Colour Doppler of Carotid Arteries. Asian J. Med. Radiol. Res. 2019;7(1):92-96.

DOI: dx.doi.org/10.21276/ajmrr.2019.7.1.20

Source of Support: Nil, Conflict of Interest: None declared. 